\title{
Scaling of Decoherence Effects in Quantum Computers
}

\author{
B. J. DALTON $\dagger \ddagger$ \\ †Centre for Atom Optics and Ultrafast Spectroscopy, \\ Swinburne University, Hawthorn, Victoria 3122, \\ Australia \\ $\ddagger$ Department of Physics, University of Queensland, \\ St Lucia, Queensland 4072, Australia
}

December 12, 2018

\begin{abstract}
The scaling of decoherence rates with qubit number $\mathrm{N}$ is studied for a simple model of a quantum computer in the situation where $\mathrm{N}$ is large. The two state qubits are localised around well-separated positions via trapping potentials and vibrational centre of mass motion of the qubits occurs. Coherent one and two qubit gating processes are controlled by external classical fields and facilitated by a cavity mode ancilla. Decoherence due to qubit coupling to a bath of spontaneous modes, cavity decay modes and to the vibrational modes is treated. A non-Markovian treatment of the short time behaviour of the fidelity is presented, and expressions for the characteristic decoherence time scales obtained for the case where the qubit/cavity mode ancilla is in a pure state and the baths are in thermal states. Specific results are given for the case where the cavity mode is in the vacuum state and gating processes are absent and the qubits are in (a) the Hadamard state (b) the GHZ state.
\end{abstract}

\section{$1 \subset$ Introduction}

Quantum computers are expected to have a key advantage over classical computers in terms of computational complexity, an advantage based on the idealised features of parallelism, entanglement and unitary quantum computation processes. For certain algorithms [1, 2], the number of computational steps needed using quantum computers should increase much more slowly with input number size than for classical computers, enabling computations that were infeasible using classical computers to be carried out. In the case of the quantum search algorithm for example, the number of steps increases with the square root of the number of items, rather than linearly as in classical searching [2]. However, the physical system whose quantum states define the $\mathrm{N}$ qubit system and the 
quantum devices involved in the gating processes both interact with the environment. Such interactions change the density operator describing the quantum computer state from a pure to a mixed state, with coherences between states evolved from different input states being partially or completely destroyed. This process of decoherence is the enemy of quantum computation, though methods such as quantum error correction [3, 4, decoherence free sub-spaces [5, 6, 7, 8] and dynamical suppression of decoherence [9, 10] could be used to minimize its effects. Utilizing the computational complexity advantage of quantum computers requires suitably large qubit numbers (ca $10^{5}$ qubits may be needed for searching or factoring algorithms with error correction [11] for input numbers of practical importance), so the scaling with the number of qubits of the decoherence rates in comparison to the coherent processing rates is important in determining the size limits of useful quantum computers [12] 13.

This paper extends previous work [14, 15, 16, 17, 18, 19, 20, treating scaling effects. Decoherence effects are studied for a simple model of an $N$ qubit quantum computer, involving $N$ two state qubit systems (see figure 1). The qubits are localised around well-separated positions via trapping potentials, and the centre of mass $(\mathrm{CM})$ vibrational motions of the qubits are treated. Coherent one and two qubit gating processes are controlled by time dependent localised classical electromagnetic (EM) fields and magnetic fields that address specific qubits. The two qubit gating processes are facilitated by a cavity mode ancilla, which permits state interchange between qubits. The magnetic fields are used to bring specific qubits into resonance with the classical EM fields or the cavity mode. The two state qubits are coupled to a bath of EM field spontaneous emission (SE) modes, and the cavity mode is coupled to a bath of cavity decay modes. For large $N$ the numerous vibrational modes of the qubits also act as a reservoir, coupled to the qubits, the cavity mode and the SE modes. The system-environment coupling interactions include electric dipole coupling of the qubits to the SE modes, quasi-mode coupling of the cavity mode to cavity decay EM field modes and Lamb-Dicke coupling of qubits to CM vibrational modes and each of: (a) the SE modes (b) the cavity mode (c) the classical EM gating fields (d) the classical magnetic gating field. The system-environment coupling interactions considered include both $\sigma_{Z}$ phase destroying and $\sigma_{X}$ population destroying terms, though the latter are more important in this model. The model is similar to those of the ion-trap 21, neutral atom 22] and cavity QED 23. 24, 25] varieties.

The object is to study decoherence effects in quantum computers for the situation where the number of qubits $N$ becomes large. Decoherence effects will be specified by the fidelity, which measures how close is the actual behaviour of the density operator for the qubits system (including the ancilla) to its idealised behaviour due to coherent gating processes only. The initial quantum state for the quantum computer will be assumed to be pure and the reservoir states will be assumed to be thermal. Previous work 14, 15, 16, 17, 18, 19, 20] indicates that the decoherence time $t_{D}$ can decrease inversely with $N$ (qubits decohering independently) or $N^{2}$ (qubits decohering collectively), so it can become very short for the case where $N$ is large and may become comparable to the reservoir 
correlation time $t_{C}$. In the case of atomic spontaneous emission relaxation, $t_{C}$ $\sim 10^{-17} \mathrm{~s}$, and $t_{D} \sim 10^{-8} \mathrm{~s}$ for one qubit could become $t_{D} \sim 10^{-18} \mathrm{~s}$ for $10^{5}$ qubits in the case of collective decoherence. However, one aspect of the present study is whether the qubits decohere independently or not, so we do not arbitrarily assume either case. As maintaining coherence between all states of the quantum computer is important for its operation, we are interested in the behaviour of all density matrix elements, and not just those for a smaller density matrix describing a single qubit, even though the latter may have long decoherence times and satisfy Markovian equations. Treating the density operator for the full $N$ qubit system via the standard Born-Markoff master equation (see for example, 26, 27]) requires that all the interaction picture density matrix elements do not change significantly during the reservoir correlation times. As the latter are just the time scales over which two-time correlation functions of reservoir operators involved in the system-reservoir interaction decay to zero, we can see that the reservoir correlation times are independent of the number of qubits. Since the general decrease in decoherence time with increasing qubit numbers indicates that some density matrix elements must change over shorter and shorter time scales, then using Markovian master equations for studying decoherence therefore becomes questionable if the number of qubits becomes large enough In the present paper we do not assume that Markovian behaviour will necessarily occur, and use methods that do not rely on the standard BornMarkoff master equation. If the behaviour does turn out to be Markovian then our approach is still correct. However as we will see, in certain cases the behaviour is not consistent with the Markoff approximation, so the necessity for our choice of non-Markovian methods is justified a posteriori. Several authors [28, 29, 30, 31] have also emphasised the importance of studying the short time regime for macroscopic and mesoscopic systems (such as quantum computers with large qubit numbers) using non-Markovian methods. One such approach [29 30 involves using eigenstates of the system operators involved in the systemreservoir interaction (pointer basis), another treatment 32] develops short-time expressions for the linear entropy (or impurity). Here the approach used is similar to that in [33] and uses the Liouville-von Neumann equations to obtain short-time expansions for the fidelity. This leads to expressions for the characteristic decoherence time scales for the general case with gating EM and magnetic fields present, and at non-zero temperature, and with the qubit and ancilla system in an arbitrary pure state. The treatment enables situations where independent or collective decoherence occur to be distinguished. In this initial paper, the decoherence time scales will be evaluated for specific initial quantum computer states such as: (a) the equal superposition of all qubit states (Hadamard state) and (b) the generalised GHZ states, with the cavity mode ancilla in the vacuum state and gating processes ignored. The effects of gating processes and the relationship of the results to those obtainable for longer times using Markovian master equation methods will be treated later.

In Section 2 the general theory used will be outlined. Results for the decoherence time scales are presented in Section 3 and Section 4 contains a summary of the paper. 


\section{Theory}

\subsection{Hamiltonian}

The total Hamiltonian is written as the sum:

$$
H=H_{S}+H_{C}+H_{B}+V_{S}+V_{I}
$$

The Hamiltonian for the qubit system and cavity mode ancilla is given in terms of qubit atomic spin operators $\sigma_{Z}^{i}$ and cavity mode annihilation, creation operators $b, b^{\dagger}$ by:

$$
H_{S}=\sum_{i} \frac{1}{2} \hbar \omega_{0} \sigma_{Z}^{i}+\hbar \omega_{b} b^{\dagger} b
$$

Here the qubits all have transition frequency $\omega_{0}$ and $\omega_{b}$ is the cavity mode frequency. The cavity mode facilitates state transfer between different qubits in two qubit gating process. In the case of a large number of charged qubits, the CM vibrational modes are too closely spaced to enable the in-phase vibrational mode to be used in this role, as in the case of ion-trap quantum computers [21. The array of qubits confined in their trapping potentials is rather analogous to a low density solid. Also, because the cavity mode energy quantum is large, this ancilla is not subject to thermal effects as are the low frequency CM vibrational modes.

The Hamiltonian for the centre of mass vibrational motion of the qubit system is given in the harmonic approximation as:

$$
\begin{aligned}
H_{C} & =\frac{1}{2 m} \sum_{i \alpha} p_{i \alpha}^{2}+\frac{1}{2} \sum_{i j \alpha \beta} V_{i j}^{\alpha \beta} \delta r_{i \alpha} \delta r_{j \beta} \\
& =\sum_{K} \hbar \nu_{K} A_{K}^{\dagger} A_{K}
\end{aligned}
$$

In these equations the qubit CM momentum operators are $p_{i \alpha}$ and the centre of mass of each qubit vibrates with displacements $\delta r_{i \alpha}$ about well-separated positions $\mathbf{r}_{i 0}$ defined by trapping potentials $(\alpha, \beta=x, y, z)$. The vibrational

mode $K$ has angular frequency $\nu_{K}$ and phonon annihilation, creation operators $A_{K}, A_{K}^{\dagger}$. The vibrational Hamiltonian $H_{C}$ applies to the general case where the qubits vibrate collectively, such as in the ion-trap case where the charged qubits interact via long-range Coulomb potentials. If the qubits vibrate independently, such as for neutral qubits with dipole-dipole couplings ignored, then the coupling matrix is given by $V_{i j}^{\alpha \beta}=\delta_{i j} \delta_{\alpha \beta} V$, and the vibrational modes $K$ are specified $i \alpha$. These all have the same frequency $\nu$.

The Hamiltonian for the bath of spontaneous emission and cavity decay modes is of the form:

$$
H_{B}=\sum_{k} \hbar \omega_{k} a_{k}^{\dagger} a_{k}+\sum_{k} \hbar \xi_{k} b_{k}^{\dagger} b_{k}
$$


Here the SE mode $k$ has angular frequency $\omega_{k}$ and annihilation, creation operators $a_{k}, a_{k}^{\dagger}$, and the cavity decay mode $k$ has angular frequency $\xi_{k}$ and annihilation, creation operators $b_{k}, b_{k}^{\dagger}$. These modes are described in the quasi-mode picture 34 .

The coherent coupling (or gating) processes for the qubit system are described by the Hamiltonian:

$$
\begin{aligned}
V_{S}= & \sum_{i}\left(\hbar \Omega_{i} \sigma_{X}^{i}+H c\right)+\sum_{i} \frac{1}{2} \hbar\left(\Delta_{i 1}-\Delta_{i 0}\right) \sigma_{Z}^{i} \\
& +\sum_{i}\left(\hbar g_{i} \sigma_{X}^{i} b+H c\right)
\end{aligned}
$$

In order, the terms are the coupling of the qubits to the classical EM field, to the magnetic field and to the cavity mode. Each qubit can be addressed by localised classical EM fields $\mathbf{E}$ and magnetic fields $\mathbf{B}$ to facilitate one and two qubit gating processes. The two qubit gating process involves the creation and annihilation of a single photon in the cavity mode, which acts as an ancilla. The quantities $\Omega_{i}, \Delta_{i a}$ and $g_{i}$ are coupling constants. The coupling constants $\Omega_{i}, \Delta_{i a}$ are both spatially localised and time dependent in accordance with the sequence of gating processes involved for a particular algorithm.

The interaction of the qubit system and ancilla with the baths and the qubits centre of mass vibrational degrees of freedom is given by:

$$
\begin{aligned}
V_{I}= & \sum_{i k}\left(\hbar g_{k}^{i} \sigma_{X}^{i} a_{k}+H c\right)+\sum_{k}\left(\hbar b^{\dagger}\left(u_{k} b_{k}+w_{k} b_{k}^{\dagger}\right)+H c\right) \\
& +\sum_{i k K}\left(\hbar n_{k K}^{i} \sigma_{X}^{i} a_{k}\left[A_{K}+A_{K}^{\dagger}\right]+H c\right) \\
& +\sum_{i K}\left(\hbar p_{K}^{i} \sigma_{X}^{i} b\left[A_{K}+A_{K}^{\dagger}\right]+H c\right) \\
& +\sum_{i K}\left(\hbar \Theta_{K}^{i} \sigma_{X}^{i}\left[A_{K}+A_{K}^{\dagger}\right]+H c\right) \\
& +\sum_{i K} \frac{1}{2} \hbar\left(m_{0 K}^{i}\left(1-\sigma_{Z}^{i}\right)+m_{1 K}^{i}\left(1+\sigma_{Z}^{i}\right)\right)\left[A_{K}+A_{K}^{\dagger}\right]
\end{aligned}
$$

The first two terms are the electric dipole coupling of the qubits to SE modes and the quasi-mode coupling of the cavity mode to the cavity decay modes. These terms involve coupling constants $g_{k}^{i}, u_{k}$ and $w_{k}$. The remaining terms are Lamb-Dicke couplings with the various fields, allowing for the vibrational motion of the qubits $\mathrm{CM}$ around their equilibrium positions $\mathbf{r}_{i 0}$. The third term allows for coupling to the SE modes, the fourth to the cavity mode, the fifth to the classical EM field and the sixth to the magnetic field. These terms involve coupling constants $n_{k K}^{i}, p_{K}^{i}, \Theta_{K}^{i}$ and $m_{0 K}^{i}$. Since this paper studies short time scale effects, the standard rotating wave approximation (RWA) has not been made. Other possible interactions, such as those due to Roentgen currents [35, 36] for neutral and charged qubit systems, and those due to ionic 
currents [35, 36] for charged qubit systems, were also investigated but found to be relatively small in comparison to the interactions listed above.

\subsubsection{Expressions}

Detailed definitions or commutation rules for the qubit, cavity mode, CM vibrational modes and bath modes operators are as follows:

$$
\begin{aligned}
\sigma_{X}^{i} & =(|1\rangle\langle 0|+| 0\rangle\langle 1|)_{i} \quad \sigma_{Y}^{i}=-i(|1\rangle\langle 0|-| 0\rangle\langle 1|)_{i} \\
\sigma_{Z}^{i} & =(|1\rangle\langle 1|-| 0\rangle\langle 0|)_{i} \\
{\left[b, b^{\dagger}\right] } & =1 \quad\left[A_{K}, A_{L}^{\dagger}\right]=\delta_{K L} \\
{\left[a_{k}, a_{l}^{\dagger}\right] } & =\delta_{k l} \quad\left[b_{k}, b_{k}^{\dagger}\right]=\delta_{k l}
\end{aligned}
$$

The qubits are represented by Pauli spin operators, whereas bosonic annihilation and creation operators apply for the cavity mode (quantized in volume $V_{b}$ ), the $\mathrm{CM}$ vibrational modes, the SE modes and the cavity decay modes (quantized in volume $V$ ).

The CM displacements $\delta r_{i \alpha}(\alpha=x, y, z)$ are related to the vibrational normal coordinates via a unitary matrix $S$ in the form:

$$
\delta r_{i \alpha}=\sum_{K} S_{i \alpha ; K} \sqrt{\frac{\hbar}{2 m \nu_{K}}}\left(A_{K}+A_{K}^{\dagger}\right),
$$

where the matrix $S$ is determined from the eigenvalue equations

$$
\sum_{j \beta} V_{i j}^{\alpha \beta} S_{j \beta ; K}=m \nu_{K}^{2} S_{i \alpha ; K}
$$

These equations also give the vibrational frequencies $\nu_{K}$. For the case where the qubits vibrate independently $S_{i \alpha ; K}=\delta_{i \alpha ; K}$ and $\nu=(V / m)^{\frac{1}{2}}$. 
Explicit expressions for the coupling constants are:

$$
\begin{aligned}
\Omega_{i}= & -i \sum_{c} \sqrt{\frac{\omega_{c}}{2 \epsilon_{0} \hbar V}}\left(\mathbf{d}_{10} \cdot \mathbf{e}_{c}\right) \alpha_{c} \exp i\left(\mathbf{k}_{c} \cdot \mathbf{r}_{i 0}-\omega_{c} t\right) \\
\Delta_{i a}= & -\left[\mathbf{m}_{a a} \cdot \mathbf{B}\right] / \hbar \quad a=0,1 \\
g_{i}= & -i \sqrt{\frac{\omega_{b}}{2 \epsilon_{0} \hbar V_{b}}}\left(\mathbf{d}_{10} \cdot \mathbf{e}_{b}\right) \exp \left(i \mathbf{k}_{b} \cdot \mathbf{r}_{i 0}\right) \\
g_{k}^{i}= & -i \sqrt{\frac{\omega_{k}}{2 \epsilon_{0} \hbar V}}\left(\mathbf{d}_{10} \cdot \mathbf{e}_{k}\right) \exp \left(i \mathbf{k} \cdot \mathbf{r}_{i 0}\right) \\
n_{k K}^{i}= & \sqrt{\frac{\omega_{k}}{2 \epsilon_{0} \hbar V}} \sqrt{\frac{\hbar}{2 m \nu_{K}}}\left(\mathbf{d}_{10} \cdot \mathbf{e}_{k}\right)\left(\mathbf{k} \cdot \mathbf{S}_{i K}\right) \times \\
& \times \exp \left(i \mathbf{k} \cdot \mathbf{r}_{i 0}\right) \\
p_{K}^{i}= & \sqrt{\frac{\omega_{b}}{2 \epsilon_{0} \hbar V_{b}}} \sqrt{\frac{\hbar}{2 m \nu_{K}}}\left(\mathbf{d}_{10} \cdot \mathbf{e}_{b}\right)\left(\mathbf{k}_{b} \cdot \mathbf{S}_{i K}\right) \times \\
& \times \exp \left(i \mathbf{k}_{b} \cdot \mathbf{r}_{i 0}\right) \\
\Theta_{K}^{i}= & \sum_{c} \sqrt{\frac{\omega_{c}}{2 \epsilon_{0} \hbar V} \sqrt{\frac{\hbar}{2 m \nu_{K}}}\left(\mathbf{d}_{10} \cdot \mathbf{e}_{c}\right)\left(\mathbf{k}_{c} \cdot \mathbf{S}_{i K}\right) \times} \\
& \times \alpha_{c} \exp i\left(\mathbf{k}_{c} \cdot \mathbf{r}_{i 0}-\omega_{c} t\right) \\
m_{a K}^{i}= & -\sum_{\beta}\left(\frac{\partial\left(\mathbf{m}_{a a} \cdot \mathbf{B}\right)}{\partial R_{i \beta}}\right)_{0} \sqrt{\frac{\hbar}{2 m \nu_{K}}} S_{i \beta ; K} / \hbar \quad a=0,1 .
\end{aligned}
$$

Here $\mathbf{d}_{10}$ is the electric dipole matrix element, $\mathbf{m}_{a a}(a=0,1)$ are the magnetic dipole matrix elements for each qubit. The polarisation unit vectors for the classical field, the cavity mode, the SE mode or cavity decay mode $k$ are $\mathbf{e}_{c}, \mathbf{e}_{b}, \mathbf{e}_{k}$ respectively, and $\mathbf{k}_{c}, \mathbf{k}_{b}, \mathbf{k}$ give the wave vectors. The classical EM gating field could be thought of as being represented by a multi-mode coherent state, where $\alpha_{c}$ is the coherent state amplitude for the mode $c$. All the modes have essentially the same polarisation vector, wave vector and frequency, though the frequency and wave vector bandwidths are sufficiently wide to produce the required spatiotemporal localisation. The equivalent classical electric field at the equilibrium position of the $i$ qubit is given by $\mathbf{E}=i \sum_{c} \sqrt{\frac{\hbar \omega_{c}}{2 \epsilon_{0} V}} \mathbf{e}_{c} \alpha_{c} \exp i\left(\mathbf{k}_{c} \cdot \mathbf{r}_{i 0}-\omega_{c} t\right)+c c$.

\subsection{Dynamics}

The total density operator $W$ for the full system of qubits, qubits CM motion, cavity mode and baths satisfies the Liouville-von Neumann equation:

$$
i \hbar \frac{\partial W}{\partial t}=[H, W]
$$

Initial conditions are assumed of an uncorrelated state for qubits and ancilla, bath and CM vibrational motion given by:.

$$
W(0)=\rho_{S}(0) \rho_{B}(0) \rho_{C}(0)
$$


and for the bath and $\mathrm{CM}$ in thermal states the average value of $V_{I}$ is zero.

$$
\operatorname{Tr}_{B C} V_{I} \rho_{B}(0) \rho_{C}(0)=\operatorname{Tr}_{B C} \rho_{B}(0) \rho_{C}(0) V_{I}=0
$$

In the present paper the initial state for the qubits and ancilla will be assumed in a pure state $\left|\psi_{S}\right\rangle$, so that $\rho_{S}(0)=\left|\psi_{S}\right\rangle\left\langle\psi_{S}\right|$.

The dynamics of the quantum computer is described by the reduced density operator $\rho_{S}$ for the qubits and ancilla subsystem defined by:

$$
\rho_{S}=\operatorname{Tr}_{B C} W
$$

The exact evolution allowing for the coherent coupling and the interaction with the bath and the CM vibrational modes is embodied in the time dependence of $\rho_{S}$. No assumption is made here that $\rho_{S}$ satisfies a Markovian master equation, though it may do so for time scales which are large compared to bath correlation times.

The coherent evolution of the qubits and ancilla subsystem is described by the reduced density operator $\rho_{S 0}$, which is chosen to coincide with the exact reduced density operator at time zero, and is then allowed to evolve under the coherent coupling term $V_{S}$ only. Hence:

$$
\begin{aligned}
i \hbar \frac{\partial \rho_{S 0}}{\partial t} & =\left[H_{S}+V_{S}, \rho_{S 0}\right] \\
\rho_{S 0}(0) & =\rho_{S}(0)
\end{aligned}
$$

Thus the interaction between the qubits/ancilla and the baths and CM motion is considered to be switched off here, hence $\rho_{S 0}$ evolves according to a Liouvillevon Neumann equation..

\subsection{Decoherence}

Decoherence effects are specified by the fidelity, defined as:

$$
F=\operatorname{Tr}_{S}\left(\rho_{S 0} \rho_{S}\right)
$$

The fidelity specifies how close the actual evolution of qubits and ancilla is to the idealised coherent evolution. The time dependence of the fidelity is entirely due to the decoherence effects caused by the bath and CM interactions with the qubits and cavity mode ancilla.

As in 33 the short-time behaviour of the fidelity with the qubits in a pure state can be expressed as a power series in the time elapsed and explicit expres- 
sions obtained for the first few time constants involved. Thus:

$$
\begin{aligned}
F(t)= & 1-\left(\frac{t}{\tau_{1}}\right)-\left(\frac{t^{2}}{2 \tau_{2}^{2}}\right)+. . \\
\frac{\hbar}{\tau_{1}}= & 0 \\
\frac{\hbar^{2}}{2 \tau_{2}^{2}}= & \operatorname{Tr}_{B C}\left\langle\psi_{S}\left|V_{I}(0)^{2}\right| \psi_{S}\right\rangle \rho_{B}(0) \rho_{C}(0) \\
& -\operatorname{Tr}_{B C}\left\langle\psi_{S}\left|V_{I}(0)\right| \psi_{S}\right\rangle^{2} \rho_{B}(0) \rho_{C}(0) \\
\equiv & \left\langle\left\langle\Delta V_{I}(0)^{2}\right\rangle_{S}\right\rangle_{B C}
\end{aligned}
$$

The initial value of the fidelity is unity. The times $\tau_{1}, \tau_{2}, .$. specify characteristic decoherence times for the qubit and ancilla system, their inverses defining decoherence rates. For thermal bath and CM states, only $\tau_{2}$ is involved in specifying short time decoherence. For the time constant $\tau_{2}$, the expression involves the average of the square of the fluctuation $\Delta V_{I}(0)=V_{I}(0)-\left\langle V_{I}(0)\right\rangle_{S}$ of the zero time interaction operator $V_{I}(0)$. The squared fluctuation operator is first averaged over the initial qubit and ancilla pure state $\left|\psi_{S}\right\rangle$, then the result is averaged over the bath and CM initial state $\rho_{B}(0) \rho_{C}(0)$.

\section{Results}

\subsection{General case}

The evaluation of $\tau_{2}$ for the general case with gating EM and magnetic fields both present, and at non-zero temperature, and with the qubit and ancilla system in an arbitrary pure state, leads to expressions for $\tau_{2}$ that involve:

(a) Expectation values for the state $\left|\psi_{S}\right\rangle$ of one qubit operators $\sigma_{\alpha}^{i}$, two qubit operators $\sigma_{\alpha}^{i} \sigma_{\beta}^{j}(i \neq j),(\alpha, \beta=X, Z)$.

(b) Expectation values of cavity operators $b, b^{\dagger}, b b^{\dagger}, b^{\dagger} b, b^{2}, b^{\dagger 2}$.

(c) Expectation values of products of these qubit and cavity operators.

(d) Sums over bath modes and centre of mass vibrational modes of products of pairs of the coupling constants $g_{k}^{i}, w_{k}, n_{k K}^{i}, p_{K}^{i}, \Theta_{K}^{i}, m_{a K}^{i}$, the products being weighted by factors involving the thermally averaged photon and phonon quantum numbers $\bar{n}_{k}, \bar{m}_{k}, \bar{N}_{K}$ for the SE modes, cavity decay modes and vibrational modes. The thermally averaged quantum numbers are given by the Planck function.

The general case will be studied in further work.

\subsection{Case of spontaneous emission for stationary qubits, no cavity mode}

In this case the cavity mode is ignored, the qubits are assumed stationary so that $\mathrm{CM}$ vibrational modes are excluded (as are all Lamb-Dicke interactions), and the only coupling constants included are $g_{k}^{i}$, given by $g_{k} \exp i k \cdot r_{i 0}$. The qubit pure 
state is $\left|\phi_{Q}\right\rangle$. This special situation has been studied in 33, where temperature effects and criteria for the qubits decohering independently or collectively were examined.

For this case the decoherence time $\tau_{2}$ is given by:

$$
\begin{aligned}
\frac{1}{2 \tau_{2}^{2}} & =\sum_{i j}\left(\left\langle\sigma_{X}^{i} \sigma_{X}^{j}\right\rangle-\left\langle\sigma_{X}^{i}\right\rangle\left\langle\sigma_{X}^{j}\right\rangle\right) \sum_{k} g_{k}^{i} g_{k}^{j *}\left(2 \bar{n}_{k}+1\right) \\
& =\sum_{i j}\left\langle\Delta \sigma_{X}^{i} \Delta \sigma_{X}^{j}\right\rangle \sum_{k}\left|g_{k}\right|^{2} \cos \mathbf{k} \cdot \mathbf{d}_{i j} \operatorname{coth} \frac{\hbar \omega_{k}}{2 k_{B} T}
\end{aligned}
$$

where $\langle.\rangle=.\left\langle\phi_{Q}|..| \phi_{Q}\right\rangle, \Delta \Omega=\Omega-\langle\Omega\rangle$ and $\mathbf{d}_{i j}=\mathbf{r}_{i 0}-\mathbf{r}_{j 0}$. This result is the same as in 33 .

As in 33 , if $\Delta k$ is the bandwidth and $\bar{k}$ the mean of a normalised Gaussian model for the function $\left|g_{k}\right|^{2} \operatorname{coth} \frac{\hbar \omega_{k}}{2 k_{B} T}$, then the condition for independent decoherence (where only terms with $i=j$ contribute to $\tau_{2}$ ) requires that $\Delta k d_{i j} \gg 1$ for any pair of qubits $\left(d_{i j}=\left|\mathbf{d}_{i j}\right|\right)$. Collective decoherence (where terms from all pairs $i \neq j$ of qubits contribute to $\left.\tau_{2}\right)$ requires that $\Delta k d_{i j} \ll 1$ and $\bar{k} d_{i j} \ll 1$. Further discussion about independent and collective decoherence is given below for the case of zero temperature, but based on evaluating the sum over SE modes $k$ for a three dimensional model.

\subsection{Case of no gating processes, zero temperature and cavity in vacuum state}

The case where no gating is taking place, the ancilla is back in its original no photon state $|0\rangle_{A}$ and the temperature is zero, provides a simple case of the general results, enabling the dependence on the qubit state to be examined. Here $\left|\psi_{S}\right\rangle=\left|\phi_{Q}\right\rangle|0\rangle_{A}$, where the state of the qubit system is $\left|\phi_{Q}\right\rangle$. This case is of some physical interest, since it corresponds to states produced after idealised coherent gating processes have occurred. Too rapid a decoherence for such states would be of concern for the implementation of quantum computers.

\subsubsection{Case of correlated qubit states}

$$
\begin{aligned}
\frac{1}{2 \tau_{2}^{2}}= & \sum_{i j}\left(\left\langle\sigma_{X}^{i} \sigma_{X}^{j}\right\rangle-\left\langle\sigma_{X}^{i}\right\rangle\left\langle\sigma_{X}^{j}\right\rangle\right) G_{i j} \\
& +\sum_{i j}\left\langle\sigma_{X}^{i} \sigma_{X}^{j}\right\rangle K_{i j}+L \\
G_{i j}= & \sum_{k} g_{k}^{i} g_{k}^{j *}+\sum_{k K} n_{k K}^{i} n_{k K}^{j *} \\
K_{i j}= & \sum_{K} p_{K}^{i} p_{K}^{j *} \\
L= & \sum_{k} w_{k} w_{k}^{*}
\end{aligned}
$$


For the correlated qubits case, the decoherence time depends on SE coupling constants, non-RWA cavity mode coupling constants and Lamb-Dicke coupling constants involving the cavity mode and the SE modes. Note that terms involving the same qubits $(i=j)$ and different $(i \neq j)$ qubits are involved.

\subsubsection{Case of uncorrelated qubit states}

In this case $\left\langle\sigma_{X}^{i} \sigma_{X}^{j}\right\rangle=\left\langle\sigma_{X}^{i}\right\rangle\left\langle\sigma_{X}^{j}\right\rangle$ for $i \neq j$, giving the decoherence time via:

$$
\begin{aligned}
\frac{1}{2 \tau_{2}^{2}}= & \sum_{i}\left(1-\left|\left\langle\sigma_{X}^{i}\right\rangle\right|^{2}\right)\left(G_{i i}+K_{i i}\right) \\
& +\sum_{i j}\left\langle\sigma_{X}^{i}\right\rangle\left\langle\sigma_{X}^{j}\right\rangle^{*} K_{i j}+L \\
= & \sum_{i}\left(1-\left|\left\langle\sigma_{X}^{i}\right\rangle\right|^{2}\right)\left(\sum_{k}\left|g_{k}^{i}\right|^{2}+\sum_{k K}\left|n_{k K}^{i}\right|^{2}+\sum_{K}\left|p_{K}^{i}\right|^{2}\right) \\
& +\left|\sum_{i}\left\langle\sigma_{X}^{i}\right\rangle \sum_{K} p_{K}^{i}\right|^{2}+L
\end{aligned}
$$

For the uncorrelated qubits case, the decoherence time depends on the same coupling constants as for the correlated case, but now only diagonal terms involving the same qubits are involved.

\subsubsection{Special sub-case of Hadamard or fiducial state}

This particular uncorrelated state results from applying the Hadamard one qubit gating process to every qubit, and which produces the important fiducial state of an equal superposition of states representing all numbers from 0 to $2^{N}-1$. The state vector for the $i$ qubit is $\left|\phi_{Q}\right\rangle_{i}=\left(|0\rangle_{i}+|1\rangle_{i}\right) / 2^{1 / 2}$. In this case the decoherence time is given by:

$$
\begin{aligned}
\frac{1}{2 \tau_{2}^{2}} & =\sum_{i j K} p_{K}^{i} p_{K}^{j *}+\sum_{k}\left|w_{k}\right|^{2} \\
\sum_{i j K} p_{K}^{i} p_{K}^{j *} & \approx N \eta^{2} g_{b}^{2}
\end{aligned}
$$

The first term is associated with the characteristic time scale due to the LambDicke term coupling the qubits, cavity mode and vibrational motion. For the general case of collective qubit vibrations, this term is only evaluated approximately, the $1 / \nu_{K}$ factor appearing in the sum over $K$ being replaced by an average value, and thus enabling the unitary properties of the matrix $S$ to be employed. Only $i=j$ terms then result. For the case of independent qubit vibrations, result (40) is exact. The Lamb-Dicke parameter $\eta \approx \mathbf{k} \cdot \boldsymbol{\delta} \mathbf{r}$ is small compared to unity. $g_{b} \approx\left(\frac{\omega_{b}}{2 \epsilon_{0} \hbar V_{b}}\right)^{1 / 2}\left|\mathbf{d}_{10}\right|$ is the cavity vacuum Rabi frequency. The second term is associated with the characteristic time scale due to the non-RWA term coupling the cavity mode and cavity decay modes. Ignoring 
this term, the decoherence time scales as $1 / \sqrt{N}$ for the Hadamard state. For $g_{b} \approx 50 \mathrm{Mhz}, \eta \approx 10^{-2}$ and thus $\eta g_{b} \approx 10^{6} \mathrm{~s}^{-1}$, we find that the decoherence time scale is about $10^{-8} \mathrm{~S}$ for $N \approx 10^{4}$ qubits. If the quantum computer were to become truly macroscopic with $N \approx 10^{22}$, the decoherence time is about $10^{-17} \mathrm{~s}$. Decoherence effects associated with the SE modes do not occur for this state, as can also been seen from the expressions for the case of stationary qubits.

\subsubsection{Special sub-case of GHZ state}

This correlated state is of special interest due to its highly entangled nature. The state vector for the qubit system is $\left|\phi_{Q}\right\rangle=(|00 . .0\rangle+|11 . .1\rangle) / 2^{1 / 2}$ and the decoherence time obtained from:

$$
\begin{aligned}
\frac{1}{2 \tau_{2}^{2}}= & \sum_{i k}\left|g_{k}^{i}\right|^{2}+\sum_{i k K}\left|n_{k K}^{i}\right|^{2} \\
& +\sum_{i K}\left|p_{K}^{i}\right|^{2}+\sum_{k}\left|w_{k}\right|^{2} \\
\sum_{i k}\left|g_{k}^{i}\right|^{2}= & N \sum_{k}\left|g_{k}\right|^{2} \\
\sum_{i k K}\left|n_{k K}^{i}\right|^{2} \approx & N \eta^{2} \sum_{k}\left|g_{k}\right|^{2} \\
\sum_{i K}\left|p_{K}^{i}\right|^{2} \approx & N \eta^{2} g_{b}^{2}
\end{aligned}
$$

The first term in the expression for $\tau_{2}$ is associated with the characteristic time scale due to the electric dipole term coupling the qubits and SE modes. It can be expressed in terms of the RMS vacuum electric field $\mathbf{E}_{r m s}$ for the SE modes as proportional to the square of the Rabi frequency $\left(\mathbf{E}_{r m s} \cdot \mathbf{d}_{10} / \hbar\right)$ associated with $\mathbf{E}_{r m s}$. The vacuum Rabi frequency for the $k$ SE mode is $g_{k} \approx\left(\frac{\omega_{k}}{2 \epsilon_{0} \hbar V_{k}}\right)^{1 / 2}\left|\mathbf{d}_{10}\right|$. The sum $\sum_{k}\left|g_{k}\right|^{2}$ can be evaluated for SE modes in three dimensions by incorporating a cut-off factor for $\left|g_{k}\right|^{2}$ of the form $\exp \left(-\omega_{k} / \omega_{c}\right)$, where $\omega_{c}$ is cut-off frequency. The correlation time for the bath of SE modes is given by the inverse of the cut-off frequency and is about $10^{-17} \mathrm{~s}$. We find that:

$$
\sum_{k}\left|g_{k}\right|^{2} \approx \Gamma \omega_{c}\left(\omega_{c} / \omega_{0}\right)^{3}
$$

Here $\Gamma$ is the SE decay rate. For $\Gamma \approx 10^{8} \mathrm{~s}^{-1}, \omega_{0} \approx 10^{15} \mathrm{~s}^{-1}, \omega_{c} \approx 10^{17} \mathrm{~s}^{-1}$ we find that $\left(\sum_{k}\left|g_{k}\right|^{2}\right)^{1 / 2} \approx 10^{15} \mathrm{~s}^{-1}$. The second term in the expression for $\tau_{2}$ is associated with the characteristic time scale due to the Lamb-Dicke term coupling the qubits, the SE modes and the CM vibrational motion. For the general case of collective qubit vibrations, this term is only evaluated approximately. For the case of independent qubit vibrations, the result is exact. As the LambDicke parameter is small compared to unity, the second term can be ignored 
in comparison with the first. The third term in the expression for $\tau_{2}$ is associated with the characteristic time scale due to the Lamb-Dicke term coupling the qubits, the cavity mode and the CM vibrational motion. For the general case of collective qubit vibrations, this term is also only evaluated approximately. For the case of independent qubit vibrations, the result is exact. It involves the square of the Rabi frequency associated with the cavity mode. For $g_{b} \approx 50 \mathrm{Mhz}$, $\eta \approx 10^{-2}$ and thus $\eta g_{b} \approx 10^{6} \mathrm{~s}^{-1}$, so the third term is also small compared to the first. The fourth term in the expression for $\tau_{2}$ is associated with the non-RWA term coupling the cavity mode and cavity decay modes. Ignoring the last term, the decoherence time scales as $1 / \sqrt{N}$ for the GHZ state. As the first term is the most important, we see that the decoherence time scale is about $10^{-17} \mathrm{~s}$ for $N \approx 10^{4}$ qubits. If the quantum computer became macroscopic with $N \approx 10^{22}$ the decoherence time is even shorter, about $10^{-26}$ s. Evidently the GHZ state would not preserve its entanglement for very long.

\subsubsection{Case of correlated qubit states - spontaneous emission deco- herence only}

The question of whether the qubits decohere collectively or independently is now examined further. For the case of stationary-qubits at zero temperature and with no cavity mode ancilla and only the coupling to the SE modes included, the decoherence time $\tau_{2}$ is given as a special case of the previous expression as:

$$
\frac{1}{2 \tau_{2}^{2}}=\sum_{i j}\left\langle\Delta \sigma_{X}^{i} \Delta \sigma_{X}^{j}\right\rangle \sum_{k}\left|g_{k}\right|^{2} \cos \mathbf{k} \cdot \mathbf{d}_{i j}
$$

Evaluating the sum $\sum_{k}\left|g_{k}\right|^{2} \cos \mathbf{k} \cdot \mathbf{d}_{i j}$ for SE modes in three dimensions and incorporating a cut-off factor for $\left|g_{k}\right|^{2}$ of the form $\exp \left(-\omega_{k} / \omega_{c}\right)$ as before, the sum is given by the expression:

$$
\sum_{k}\left|g_{k}\right|^{2} \cos \mathbf{k} \cdot \mathbf{d}_{i j} \approx \Gamma \omega_{c}\left(\omega_{c} / \omega_{0}\right)^{3} F\left(\omega_{c} \tau_{i j}, \cos ^{2} \theta_{i j ; 10}\right)
$$

where $\tau_{i j}=d_{i j} / c$ is the time for light to travel between the $i$ and $j$ qubits, $\theta_{i j ; 10}$ is the angle between the vectors for the separation of the qubits $\mathbf{d}_{i j}$ and the dipole moment $\mathbf{d}_{10}$. The function $F$ is of order unity and involves ratios of polynomials in the quantity $\omega_{c} \tau_{i j}$. For $\omega_{c} \tau_{i j} \gg 1$ this function behaves like $\left(\omega_{c} \tau_{i j}\right)^{-4}$. This is in contrast to the one dimensional case at low temperatures treated in [33], where no such decrease for large $\omega_{c} \tau_{i j}$ occurs. For $\Gamma \approx 10^{8} \mathrm{~s}^{-1}, \omega_{0} \approx 10^{15} \mathrm{~s}^{-1}, \omega_{c} \approx 10^{17} \mathrm{~s}^{-1}$ (as above) we find that $\left(\Gamma \omega_{c}\left(\omega_{c} / \omega_{0}\right)^{3}\right)^{1 / 2} \approx 10^{15} \mathrm{~s}^{-1}$. Allowing for conveniently addressing separate qubits with optical laser fields, the nearest neighbour distance will be taken as $d_{i j} \approx 10^{-6} \mathrm{~m}$, hence $\omega_{c} \tau_{i j} \approx 10^{3} \gg 1$. Thus even for nearest neighbour qubits, $\left(\Gamma \omega_{c}\left(\omega_{c} / \omega_{0}\right)^{3} F\right)^{1 / 2} \approx 10^{9} \mathrm{~s}^{-1}$. This is a factor of $10^{6}$ smaller than for the $i=j$ terms. For more widely separated qubits the reduction is even greater. The greater number of qubits in a shell of qubits between $d_{i j}, d_{i j}+\delta d_{i j}$ (proportional to $d_{i j}^{2}$ ) does not overcome the $d_{i j}^{-4}$ factor. Thus it would seem that only the $i=j$ 
terms will be important in the expression for the decoherence time $\tau_{2}$. This indicates that the qubits decohere independently. Including then only the $i=j$ terms (which are all equal), the expression for $1 / 2 \tau_{2}^{2}$ is proportional to $N$ and is non zero for correlated states. Overall, the decoherence time scales as $1 / \sqrt{N}$ for correlated states. Similar considerations to before again lead to a decoherence time scale of about $10^{-17} \mathrm{~s}$ for $N \approx 10^{4}$ qubits, whilst for a macroscopic size quantum computer with $N \approx 10^{22}$ the decoherence time is about $10^{-26} \mathrm{~s}$. Remembering that the SE bath correlation time is of order $\omega_{c}^{-1} \approx 10^{-17} \mathrm{~s}$, then these decoherence times are not long compared to the correlation time, so nonMarkovian conditions apply as has been expected. If spontaneous emission is present this would seem to be something of a problem for quantum computation based on the model treated here, since processing involving correlated states is an essential feature.

\subsection{Case of no gating processes, zero temperature, cavity in vacuum state but spontaneous emission absent}

The case where no gating is taking place, the ancilla is back in its original no photon state $|0\rangle_{A}$, the temperature is zero, and SE modes are ignored provides a simple case of the general results, enabling the dependence on the qubit state to be examined. Here it is assumed that the cavity is of such high quality that qubit decay via the SE modes can be ignored, so the only decay is via LambDicke coupling through the cavity mode. Again $\left|\psi_{S}\right\rangle=\left|\phi_{Q}\right\rangle|0\rangle_{A}$, where the state of the qubit system is $\left|\phi_{Q}\right\rangle$. This case is of some physical interest, since it corresponds to states produced after idealised coherent gating processes have occurred, but now in a more favourable cavity situation. Too rapid decoherence of correlated qubit states - even in this case where SE decay is absent - would be of concern for the implementation of quantum computers.

\subsubsection{Case of Correlated qubit States}

The previous expressions can be used to obtain the decoherence time $\tau_{2}$ by deleting all contributions associated with the SE modes. This gives:

$$
\begin{aligned}
\frac{1}{2 \tau_{2}^{2}} & =\sum_{i j}\left\langle\sigma_{X}^{i} \sigma_{X}^{j}\right\rangle K_{i j}+L \\
K_{i j} & =\sum_{K} p_{K}^{i} p_{K}^{j *} \quad L=\sum_{k} w_{k} w_{k}^{*}
\end{aligned}
$$

\subsubsection{Evaluation of Lamb-Dicke Term}

For the general case of collective qubit vibrations, an approximate evaluation of the Lamb-Dicke term yields the result:

$$
\frac{1}{2 \tau_{2}^{2}} \approx N \eta^{2} g_{b}^{2}
$$


Result (50) is exact for the case of independent qubit vibrations. This term is associated with the characteristic time scale due to the Lamb-Dicke term coupling the qubits, cavity mode and vibrational motion. The Lamb-Dicke parameter $\eta \approx \mathbf{k} \cdot \boldsymbol{\delta} \mathbf{r}$ is small compared to unity. $g_{b} \approx\left(\frac{\omega_{b}}{2 \epsilon_{0} \hbar V_{b}}\right)^{1 / 2}\left|\mathbf{d}_{10}\right|$ is the cavity vacuum Rabi frequency. The decoherence time scales as $1 / \sqrt{N}$. For $g_{b} \approx$ $50 \mathrm{Mhz}, \eta \approx 10^{-2}$ and thus $\eta g_{b} \approx 10^{6} \mathrm{~s}^{-1}$, the decoherence time scale is found to be about $10^{-8} \mathrm{~S}$ for $N \approx 10^{4}$ qubits, whilst for a macroscopic sized quantum computer with $N \approx 10^{22}$ the decoherence time is about $10^{-17} \mathrm{~s}$. Although it might be thought that these decoherence times are rather long compared to bath correlation times, it should be noted that for the $\mathrm{CM}$ vibrational modes bath, the correlation time is of order $\nu^{-1}$, where $\nu$ is a typical CM vibrational frequency. As $\nu \approx 2 \pi \cdot 10^{6} \mathrm{~s}^{-1}$ [21, 22], the correlation time for the bath is about $10^{-6} \mathrm{~s}$. Thus these decoherence times are short compared to the correlation time, so non-Markovian conditions apply as has been expected. The first result is more promising from the point of view of implementing quantum computers of a useful size, for example with $N \approx 10^{4}$. In the general case where the qubits vibrate collectively, the evaluation is only approximate however and a full treatment will involve determining the matrix elements $S_{i \alpha ; K}$ relating qubit $\mathrm{CM}$ displacements to the vibrational normal coordinates in order to examine issues such as whether the qubits decohere independently or collectively.

\section{Summary}

Decoherence effects in quantum computers have been studied for the situation where the number of qubits $N$ becomes large. A standard model involving $N$ two state qubit systems was treated, with localised, well-separated qubits undergoing vibrational motion in the trapping potentials. Coherent one and two qubit gating processes were controlled by time dependent localised classical EM and magnetic fields, the two qubit gating processes being facilitated by a cavity mode ancilla. The qubits were coupled to bath of spontaneous emission modes, the cavity mode was coupled to a bath of cavity decay modes. The numerous vibrational qubit modes also behave as a reservoir. Non-RWA couplings and effects of qubit vibrational motion were both included. The predominant coupling of qubits to the baths is amplitude coupling via the $\sigma_{X}^{i}$ Pauli operators. Decoherence effects were specified by the fidelity, and non-Markovian expressions for the short time behaviour of the fidelity were obtained for the general case where the qubits and ancilla are in any pure state and the baths and qubit vibrational modes are in thermal states.

Characteristic decoherence time scales were evaluated for specific qubit states in the simple situation when no gating processes are occurring and at zero temperature. The decoherence time is mainly affected by the spontaneous emission decay of the qubits. Decoherence times scaling inversely as $1 / \sqrt{N}$ were found for the cases treated. For the uncorrelated Hadamard state a decoherence time scale of about $10^{-8} \mathrm{~S}$ for $N \approx 10^{4}$ qubits was found, for a macroscopic $N \approx 10^{22}$ it was about $10^{-17} \mathrm{~s}$. For the correlated GHZ state a decoherence time scale of 
about $10^{-17} \mathrm{~s}$ for $N \approx 10^{4}$ qubits was found, for a macroscopic size quantum computer with $N \approx 10^{22}$ it was about $10^{-26}$ s. Correlated states generally were found to have similar decoherence times to the GHZ state, and the qubits were found to decohere independently.

Characteristic decoherence time scales were also evaluated for specific qubit states in a further simple situation when no gating processes are occurring, the temperature is zero, and decay via spontaneous emission modes is ignored. The decoherence time is now mainly affected by the Lamb-Dicke coupling of the qubits to the cavity mode ancilla and to the CM vibrational modes. Decoherence times scaling inversely as $1 / \sqrt{N}$ for the cases treated were found. A decoherence time scale of about $10^{-8} \mathrm{~S}$ for $N \approx 10^{4}$ qubits was found, for a macroscopic size quantum computer with $N \approx 10^{22}$ it was about $10^{-17} \mathrm{~s}$.

The case where spontaneous emission decay can be disregarded, such as when the qubits are all in a very high $\mathrm{Q}$ cavity, is far more promising from the point of view of implementing quantum computers of useful size, say with $N \approx 10^{4}$ qubits. An alternative, though more complex model for avoiding spontaneous emission effects would be to replace the two internal state qubits by qubits with three internal states, such as in a lambda system. In such a model the qubit states would be two near degenerate ground states, and the upper state would be in effect part of the ancilla system and only coming into play during the gating processes. Spontaneous emission would then only be important during the gating processes and could be minimized if the latter occurred in short enough times. A similar model has been examined in a different context in [7].

Finally, although the decoherence times found here are not long compared to bath correlation times, and hence non-Markovian conditions apply as expected, it would be of interest to consider the behaviour of the fidelity in the Markovian regime for the present model in order that the short time scale results for decoherence effects presented here can be linked up with those for longer time scales.

\section{Figure caption}

Figure 1. Model of an $N$ qubit quantum computer. Two state qubits are localised around well-separated positions via trapping potentials, and undergo centre of mass $(\mathrm{CM})$ vibrational motions. Coherent one and two qubit gating processes are controlled by time dependent localised classical electromagnetic (EM) fields and magnetic fields that address specific qubits. Two qubit gating processes are facilitated by a cavity mode ancilla, which permits state interchange between qubits. Magnetic fields are used to bring specific qubits into resonance with the classical EM fields or the cavity mode. The two state qubits are coupled to a bath of EM field spontaneous emission (SE) modes, and the cavity mode is coupled to a bath of cavity decay modes. For large $N$ the nu-

merous vibrational modes of the qubits also act as a reservoir, coupled to the qubits, the cavity mode and the SE modes. 


\section{References}

[1] SHOR, P. W., 1995, Quant-Ph/9508027

[2] GROVER, L., 1997, Phys. Rev. Lett. 79, 325

[3] SHOR, P. W., 1995, Phys. Rev. A 52, R2493

[4] STEAne, A. W., 1996, Proc. Roy. Soc. A 452, 2251

[5] LIDAR, D. A., CHUANG, I. L., and WhaleY, K. B., 1998, Phys. Rev. Lett. 81, 2594.

[6] Plenio, M. B., HUElGA, S. F., BEIGE, A., and KNIGHT, P. L., 1999, Phys. Rev. A 59, 2458.

[7] BEIGE, A., BRAun, D., TREgEnNA, B., and KNIGHT, P. L., 2000, Phys. Rev. Lett. 85, 1762.

[8] JAne, E., Plenio, M. B., and JOnAthon, D., 2002, Phys. Rev. A 65, 050302 .

[9] VIOLA, L., and LlOYD, S., 1998, Phys. Rev. A 58, 2733.

[10] GEA-BANACLOCHE, J., 2001, J. Mod. Opt. 48, 927.

[11] PRESKILL, J., 1999, Physics Today, p24, June.

[12] PLEniO, M., and KNIGHT, P. L., 1996, Phys. Rev. A 53, 2986.

[13] PleniO, M., and KNIGHT, P. L., 1997, Proc. Roy. Soc. A 453, 2017.

[14] UNRUH, W. G., 1995, Phys. Rev. A 51, 992.

[15] PAlma, G. M., SUOMINEN, K.-A., and EKERT, A. K., 1996, Proc. Roy. Soc. A 452, 567 .

[16] GARG, A., 1996, Phys. Rev. Lett. 77, 964.

[17] DUAN, L-M., and GUO, G-C., 1998, Phys. Rev. A 57, 737.

[18] DUAN, L-M., and GUO, G-C., 1998, Phys. Rev. A 58, 3491.

[19] DUAN, L-M., and GUO, G-C., 1998, Quant. Semiclass. Opt. 10, 611.

[20] SOREnSEn, A., and MOLMeR, K., 2000, Phys. Rev. A 62, 022311.

[21] CIRAC, J. I. and ZOLLER, P., 1995, Phys. Rev. Lett. 74, 4091.

[22] BRIEGEL, H.-J., CALCARCO, T., JAKSCH, D., CIRAC, J. I., and ZOLLER, P., 2000, J. Mod. Opt. 47, 415.

[23] DOMOKOS, P., RAIMOND, J. M., BRUNE, M., and HAROCHE, S., 1995, Phys. Rev. A 52, 3554. 
[24] PellizzARI, T., GARDiner, S. A., CIRAC, J. I., and ZOLlER, P., 1995, Phys. Rev. Lett. 75, 3788.

[25] TURChetTe, Q. A., HOOD, C. J., LANGE, W., MABUCHI, H., and KIMBLE, H. J., 1995, Phys. Rev. Lett. 75, 4710.

[26] BARNETT, S. M., and RADMORE, P. M., 1997, Methods in Theoretical Quantum Optics (Oxford, UK: Clarendon Press).

[27] DALTON, B. J., FICEK, Z., and SWAIN, S. S., 1999, J. Mod. Opt. 46, 379.

[28] UNRUH, W. G., and ZUREK, W. H., 1989, Phys. Rev. D 40, 1071

[29] BRAUn, D., HAAKE, F., and STRUnZ, W. T., 2001, Phys. Rev. Lett. 86, 2913.

[30] PRIVMAn, V., 2002, Mod. Phys. Lett. B, 16, 459.

[31] STRUNZ, W. T., HAAKE, F., and BRAUN, D., 2002, quant-ph/0204129.

[32] Retamal, J. C., and ZAGURY, N., 2001, Phys. Rev. A 63, 032106.

[33] DUAN, L-M., and GUO, G-C., 1997, Phys. Rev. A 56, 4466.

[34] DAlton, B. J., BARnETT, S. M., and KNIGHT, P. L., 1999, J. Mod Opt. 46, 1315.

[35] BAXter, C., BABIKER, M., and LOUdOn, R., 1993, Phys. Rev. A 47, 1278.

[36] WILKenS, M., 1994, Phys. Rev. A 48, 570. 

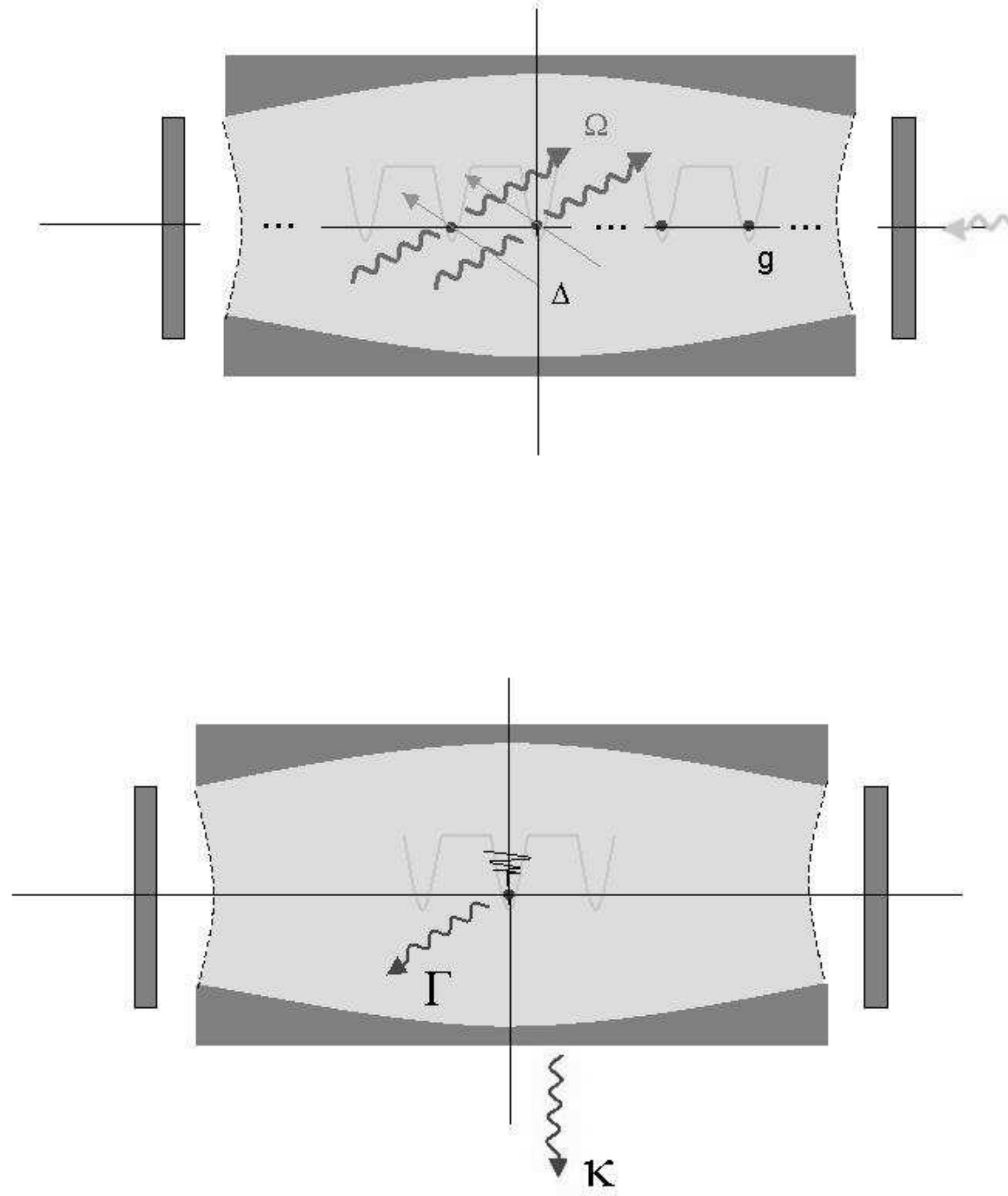